\title{
Photon Structure in Supersymmetric QCD
}

\section{Yoshio Kitadono}

Institute of Physics, Academia Sinica

Taipei, Taiwan 115, Republic of China

E-mail: kitadonodphys.sinica.edu.tw

\section{Ryo Sahara}

Department of Physics, Graduate School of Science, Kyoto University

Kitashirakawa, Kyoto 606-8502, Japan

E-mail: saharaescphys.kyoto-u.ac.jp

Tsuneo Uematsu*

Department of Physics, Graduate School of Science, Kyoto University

Kitashirakawa, Kyoto 606-8502, Japan

E-mail: uematsuescphys.kyoto-u.ac.jp

\section{Yutaka Yoshida}

High Energy Accelerator Research Organization (KEK)

1-1 Oh-ho, Tsukuba, Ibaraki 305-0801, Japan

E-mail: yyoshida@post.kek.jp

We investigate the virtual photon structure functions in the supersymmetric QCD, where we have squarks and gluinos in addition to the quarks and gluons. For the parton model, we evaluate the eight virtual photon structure functions and study the squark mass effects as well as the positivity constraints. We also calculate the heavy sparticle mass effects in the framework of DGLAP equations with the appropriate boundary conditions for the heavy particles to the leading order in SUSY QCD.

10th International Symposium on Radiative Corrections (Applications of Quantum Field Theory to Phenomenology) - Radcor2011

September 26-30, 2011

Mamallapuram, India

\footnotetext{
*Speaker.
} 


\section{Introduction}

In the present talk we would like to discuss about the virtual photon structure functions in the supersymmetric (SUSY) QCD, where squarks and gluinos are present in addition to quarks and gluons. Here we particularly interested in the heavy particle mass effects on the parton distributions inside the virtual photon in SUSY QCD.

As is well known, the photon structure functions can be studied through the two-photon processes in the $e^{+} e^{-}$collision which dominate over one-photon annihilation processes at high energies [1 2]. Here we consider the two-photon processes with double-tagging experiments where both of the outgoing $e^{+}$and $e^{-}$are detected. In particular we study the kinematical region in which one of the photons, which we call the 'probe photon', with the mass squared $-q^{2}=Q^{2}$, is much heavier than the other one, 'target photon', with the mass squared $-p^{2}=P^{2}$, which is in turn much larger than $\Lambda_{\mathrm{QCD}}^{2}$, i.e. $\Lambda_{\mathrm{QCD}}^{2} \ll P^{2} \ll Q^{2}$. In this kinematical region we can study the whole structure functions and the parton distributions, their shapes and magnitudes in perturbation theory.

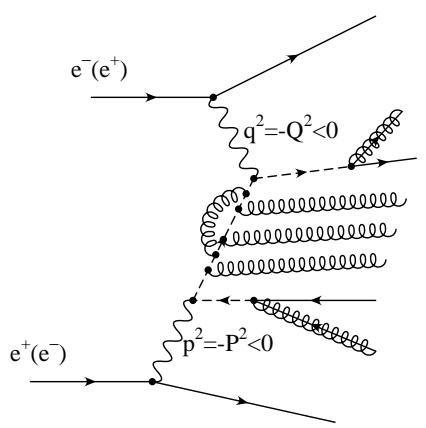

Figure 1: $e^{+} e^{-}$two-photon processes in supersymmetric $\mathrm{QCD}$. The solid (dashed) line denotes the quark (squark), while the spiral (spiral-straight) line implies the gluon (gluino).

Some years ago the effects of supersymmetry on two-photon process were studied in the literature [3] 4]. In 1983 Reya studied the SUSY effects in photon structure functions where the squark mass is less than $40 \mathrm{GeV}$ [3]. The one-loop splitting functions for SUSY QCD were computed in refs. [6]. Scott and Stirling studied the longitudinal photon structure function in SUSY QCD [8]. Ross and Weston investigated the evolution and threshold effects for the virtual photon structure functions [4]. In this paper based on the framework of treating heavy parton distributions [9 10] we reexamine the effects of the squarks and gluinos appearing in SUSY QCD (SQCD) on the photon structure functions to the leading order in SQCD which can be measured in the two-photon processes of $e^{+} e^{-}$collision illustrated in Fig 1

\section{Squark contribution to the photon structure functions}

Before going to the study of the SUSY QCD radiative effects based on the $Q^{2}$ evolution equation, it is worthwhile to investigate squark contributions to the photon structure functions through the pure QED interaction. We consider the virtual photon-photon forward scattering amplitude for $\gamma(q)+\gamma(p) \rightarrow \gamma(q)+\gamma(p)$ illustrated in Fig.2. The $s$-channel helicity amplitudes are related to its 
absorptive part as follows:

$$
W\left(a b \mid a^{\prime} b^{\prime}\right)=\varepsilon_{\mu}^{*}(a) \varepsilon_{\rho}^{*}(b) W^{\mu v \rho \sigma} \varepsilon_{v}\left(a^{\prime}\right) \varepsilon_{\sigma}\left(b^{\prime}\right),
$$

where

$$
W_{\mu v \rho \sigma}(p, q)=\frac{1}{\pi} \operatorname{Im} T_{\mu v \rho \sigma}(p, q),
$$

and $\varepsilon_{\mu}(a)$ represents the photon polarization vector with helicity $a$, and $a=0, \pm 1$. Similarly for the other polarization vectors and we have $a^{\prime}, b, b^{\prime}=0, \pm 1$. Due to the angular momentum conservation, parity conservation and time reversal invariance, we have in total eight independent $s$-channel helicity amplitudes, which we may take as

$$
\begin{aligned}
& W(1,1 \mid 1,1), W(1,-1 \mid 1,-1), W(1,0 \mid 1,0), W(0,1 \mid 0,1), W(0,0 \mid 0,0), \\
& W(1,1 \mid-1,-1), W(1,1 \mid 0,0), W(1,0 \mid 0,-1) .
\end{aligned}
$$

The first five amplitudes are helicity-nonflip and the last three are helicity-flip. It is noted that the $s$-channel helicity-nonflip amplitudes are semi-positive, but not the helicity-flip ones. The photon-

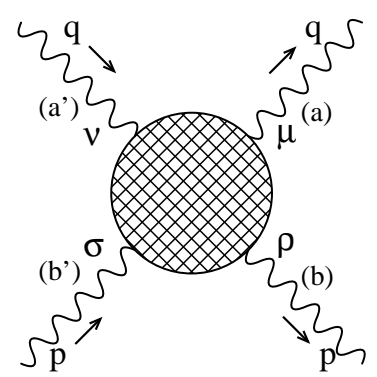

Figure 2: Virtual photon-photon forward scattering with momenta $q(p)$ and helicities $a(b)$ and $a^{\prime}\left(b^{\prime}\right)$

photon scattering phenomenology is often discussed in terms of the photon structure functions instead of the s-channel helicity amplitudes. Budnev, Chernyak and Ginzburg [BCG] [11] introduced the following eight independent structure functions, in terms of which the absorptive part of virtual photon-photon forward scattering, $W^{\mu v \rho \sigma}$, is written as,

$$
\begin{aligned}
W_{\mu v \rho \sigma}= & \left(T_{T T}\right)_{\mu v \rho \sigma} W_{T T}+\left(T_{T T}^{a}\right)_{\mu v \rho \sigma} W_{T T}^{a}+\left(T_{T T}^{\tau}\right)_{\mu v \rho \sigma} W_{T T}^{\tau}+\left(T_{L T}\right)_{\mu v \rho \sigma} W_{L T} \\
& +\left(T_{T L}\right)_{\mu v \rho \sigma} W_{T L}+\left(T_{L L}\right)_{\mu v \rho \sigma} W_{L L}-\left(T_{T L}^{\tau}\right)_{\mu v \rho \sigma} W_{T L}^{\tau}-\left(T_{T L}^{\tau a}\right)_{\mu v \rho \sigma} W_{T L}^{\tau a},
\end{aligned}
$$

where $T_{i}$ 's are the projection operators given in the Appendix of ref. [15], and these eight structure functions are simply related to the s-channel helicity amplitudes as discussed in ref. [11].

The structure functions are evaluated by multiplying the relevant projection operator to the structure tensor $W_{\mu v \rho \sigma}$. We note that the virtual photon structure functions $F_{1}^{\gamma}, F_{2}^{\gamma}, F_{L}^{\gamma}, g_{1}^{\gamma}$ and $g_{2}^{\gamma}$ are related to the ones introduced by BCG in [11] as

$$
\begin{aligned}
& F_{1}^{\gamma}\left(x, Q^{2}, P^{2}\right)=W_{T T}-\frac{1}{2} W_{T L}, \quad F_{2}^{\gamma}\left(x, Q^{2}, P^{2}\right)=\frac{x}{\widetilde{\beta}^{2}}\left[W_{T T}+W_{L T}-\frac{1}{2} W_{L L}-\frac{1}{2} W_{T L}\right], \\
& F_{L}^{\gamma}\left(x, Q^{2}, P^{2}\right)=F_{2}^{\gamma}-x F_{1}^{\gamma}, \\
& g_{1}^{\gamma}\left(x, Q^{2}, P^{2}\right)=\frac{1}{\widetilde{\beta}^{2}}\left[W_{T T}^{a}-\sqrt{1-\widetilde{\beta}^{2}} W_{T L}^{\tau a}\right], \quad g_{2}^{\gamma}\left(x, Q^{2}, P^{2}\right)=-\frac{1}{\widetilde{\beta}^{2}}\left[W_{T T}^{a}-\frac{1}{\sqrt{1-\widetilde{\beta}^{2}}} W_{T L}^{\tau a}\right],
\end{aligned}
$$


where independent variables are $x=Q^{2} / 2 p \cdot q$ (Bjorken variable), $P^{2}, Q^{2}$ and $m^{2}$. Here we have introduced the variable $\widetilde{\beta}$ given as

$$
\widetilde{\beta}=\sqrt{1-\frac{P^{2} Q^{2}}{(p \cdot q)^{2}}}=\sqrt{1-\frac{4 x^{2} P^{2}}{Q^{2}}} .
$$
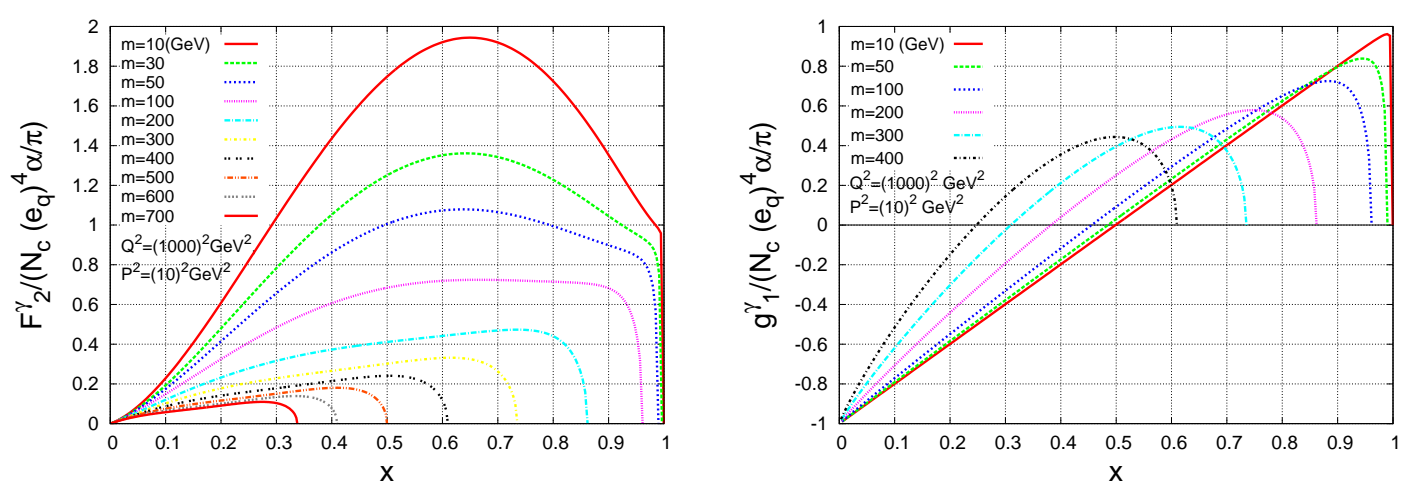

Figure 3: $F_{2}^{\gamma}$ (left) and $g_{1}^{\gamma}$ (right) as functions of $x$ for various values of squark mass, $m$, which are given in units of $\mathrm{GeV}$, for fixed $P^{2}=(10)^{2} \mathrm{GeV}^{2}$ and $Q^{2}=(1000)^{2} \mathrm{GeV}^{2}$.
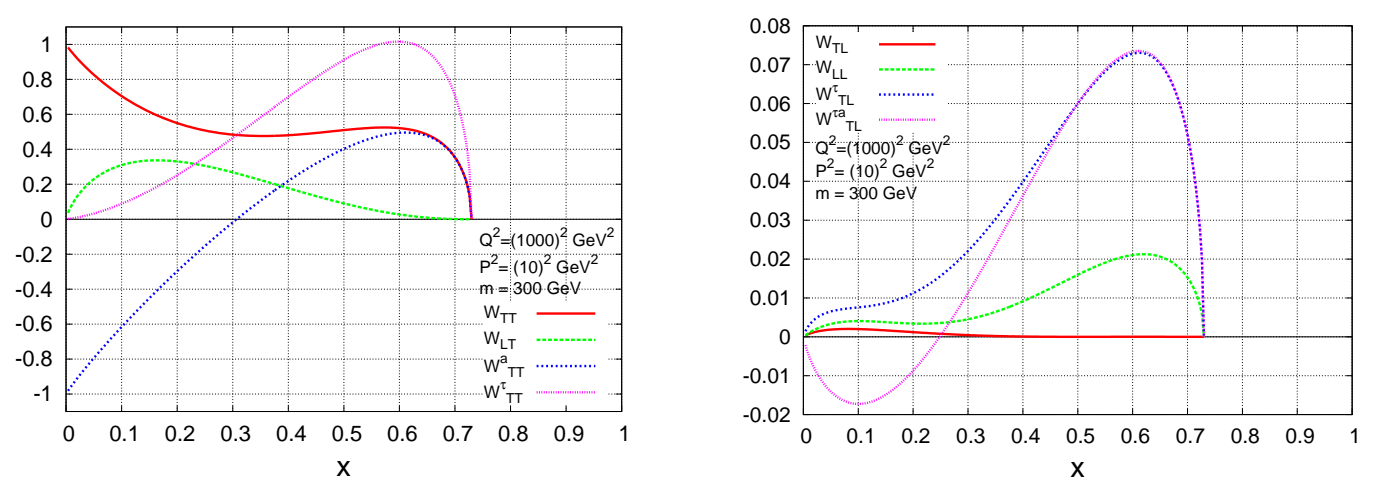

Figure 4: Eight virtual photon structure functions: $W_{T T}, W_{L T}, W_{T T}^{a}, W_{T T}^{\tau}$ (Left), $W_{T L}, W_{L L}, W_{T L}^{\tau}, W_{T L}^{\tau a}(\mathrm{Right})$. The values of $P^{2}$ and $Q^{2}$ are in units of $\mathrm{GeV}^{2}$.

We have plotted in Figs.3 and 4, squark contributions to the photon structure functions as functions of $x$. The vertical axes are in units of $N_{c} \frac{\alpha}{\pi} e_{q}^{4}$, where $N_{c}$ is the number of colors, $N_{c}=3$ for supersymmetric QCD. $e_{q}$ is the electric charge of the squark which is the super partner of the quark of the $q$-th flavor. In these plots we have chosen $Q^{2}=(1000)^{2} \mathrm{GeV}^{2}$, and $P^{2}=(10)^{2} \mathrm{GeV}^{2}$. The allowed $x$ region is $0 \leq x \leq x_{\max }$ with

$$
x_{\max }=\frac{1}{1+\frac{P^{2}}{Q^{2}}+\frac{4 m^{2}}{Q^{2}}} .
$$


The photon structure functions can be classified into two groups: (i) $W_{T T}, W_{L T}, W_{T T}^{a}, W_{T T}^{\tau}$ and (ii) $W_{T L}, W_{L L}, W_{T L}^{\tau}, W_{T L}^{\tau a}$. The first group also exists for the real photon target, while the second group does not exist for the real photon case and are small in magnitude compared to the first group. The graphs show that all the structure functions tend to vanish as $x \rightarrow x_{\max }$ which is the kinematical constraint. The positivity constraints derived from the general Cauchy-Schwarz inequalities [14] in fact lead to the following equalities for the squark contributions [15]:

$$
\begin{aligned}
& W_{T T}^{\tau}=W_{T T}+W_{T T}^{a}, \\
& \left|W_{T L}^{\tau}-W_{T L}^{\tau a}\right|=\sqrt{W_{T L} W_{L T}},
\end{aligned}
$$

while we have an inequality

$$
\left|W_{T L}^{\tau}+W_{T L}^{\tau a}\right| \leq \sqrt{\left(W_{T T}+W_{T T}^{a}\right) W_{L L}} .
$$

The first equality (2.8) can be rewritten in terms of the helicity amplitudes as

$$
W(1,1 \mid-1,-1)=W(1,1 \mid 1,1),
$$

which holds both for the real $\left(P^{2}=0\right)$ and virtual $\left(P^{2} \neq 0\right)$ photon target.

\section{Evolution equation and heavy mass effects}

Many authors have studied heavy quark mass effects in the nucleon [12] and the photon structure functions [13 14]. Now we consider the heavy parton mass effects, and we decompose the parton distributions in the case where we have $n_{f}-1$ light quarks and one heavy quark flavor which we take the $n_{f}$-th quark and all the squarks have the same heavy mass, while the gluino has another heavy mass [9]0]:

$$
\vec{q}^{\gamma}(t)=\left(G, \lambda, q_{1}, \cdots, q_{n_{f}-1}, s_{1}, \cdots, s_{n_{f}-1}, q_{H}, s_{H}\right) .
$$

We denote the $i$-th light flavor quark, squark by $q_{i}\left(x, Q^{2}, P^{2}\right), s_{i}\left(x, Q^{2}, P^{2}\right),\left(i=1, \cdots, n_{f}-1\right)$, one heavy quark and its superpartner (squark) by $q_{H}, s_{H}$ and the gluon, gluino by $G\left(x, Q^{2}, P^{2}\right)$, $\lambda\left(x, Q^{2}, P^{2}\right)$, respectively. We now define light flavor-nonsinglet (LNS) and singlet (LS) combination of the quark and the squark as follows:

$$
\begin{gathered}
q_{L N S}=\sum_{i=1}^{n_{f}-1}\left(e_{i}^{2}-\left\langle e^{2}\right\rangle_{L}\right) q_{i}, \quad s_{L N S}=\sum_{i=1}^{n_{f}-1}\left(e_{i}^{2}-\left\langle e^{2}\right\rangle_{L}\right) s_{i}, \\
q_{L S}=\sum_{i=1}^{n_{f}-1} q_{i}, \quad s_{L S}=\sum_{i=1}^{n_{f}-1} s_{i}, \quad\left\langle e^{2}\right\rangle_{L}=\frac{1}{n_{f}-1} \sum_{i=1}^{n_{f}-1} e_{i}^{2} .
\end{gathered}
$$

Then we rearrange the parton distributions as

$$
\vec{q}^{\gamma}(t)=\left(G, \lambda, q_{L S}, s_{L S}, q_{H}, s_{H}, q_{L N S}, s_{L N S}\right) .
$$

The evolution equations are given by

$$
\frac{d \vec{q}^{\gamma}(t)}{d t}=\vec{q}^{\gamma}(t) \otimes P^{(0)}+\frac{\alpha}{\alpha_{s}(t)} \vec{k}^{(0)}
$$


where the parton-parton splitting function $P^{(0)}$ and the photon-parton splitting function $\vec{k}^{(0)}$ are given in ref. [6, 16].

Now we take into account the heavy mass effects by setting the initial conditions for the heavy parton distribution functions as discussed in [10]. We note here that the structure function $F_{2}^{\gamma}$ can be written as a convolution of the parton distribution and the Wilson coefficient function $F_{2}^{\gamma}\left(x, Q^{2}, P^{2}\right) / x=\vec{q}^{\gamma} \otimes \vec{C}$. We put the initial conditions for the moments of the parton distributions are defined as

$$
\vec{q}^{\gamma}(n, t=0)=\left(0, \hat{\lambda}(n), 0, \hat{s}_{L S}(n), \hat{q}_{H}(n), \hat{s}_{H}(n), 0, \hat{s}_{L N S}(n)\right),
$$

and require that the following boundary conditions are satisfied:

$$
\begin{aligned}
& \lambda\left(n, Q^{2}=m_{\lambda}^{2}\right)=0, s_{L S}\left(n, Q^{2}=m_{s q}^{2}\right)=0, q_{H}\left(n, Q^{2}=m_{H}^{2}\right)=0, \\
& s_{H}\left(n, Q^{2}=m_{s q}^{2}\right)=0, s_{L N S}\left(n, Q^{2}=m_{s q}^{2}\right)=0,
\end{aligned}
$$

where $m_{\lambda}, m_{s q}$ and $m_{H}$ are the mass of the gluino, squarks and the heavy (here we take top) quark, respectively. Note that here we take all the squarks have the same mass $m_{s q}$.

By solving the evolution equation taking into account the above boundary condition we get for the moment of $\vec{q}^{\gamma}$ :

$$
\vec{q}^{\gamma}(n, t)=\frac{\alpha}{8 \pi \beta_{0}} \frac{4 \pi}{\alpha_{s}(t)} \vec{K}_{n}^{(0)} \sum_{i} P_{i}^{n} \frac{1}{1+d_{i}^{n}}\left\{1-\left[\frac{\alpha_{s}(t)}{\alpha_{s}(0)}\right]^{1+d_{i}^{n}}\right\}+\vec{q}^{\gamma}(n, 0) \sum_{i} P_{i}^{n}\left[\frac{\alpha_{s}(t)}{\alpha_{s}(0)}\right]^{d_{i}^{n}}
$$

where the $P_{i}^{n}$ is the projection operator onto the eigenstate $\lambda_{i}$ of the anomalous dimension matrices $\hat{\gamma}_{n}=\sum_{i} P_{i}^{n} \lambda_{i}^{n}$ which are related to the splitting function $P(x)$ as $\hat{\gamma}_{n} \equiv-2 \int_{0}^{1} d x x^{n-1} P(x)$ and $d_{i}^{n} \equiv$ $\lambda_{i}^{n} / 2 \beta_{0} . \vec{K}_{n}^{(0)}$ is the anomalous dimension corresponding to the photon-parton splitting function, $\vec{K}_{n}^{(0)}=2 \int_{0}^{1} d x x^{n-1} \vec{k}^{0}(x)$. The initial value $\vec{q}^{\gamma}(n, 0)$ is determined so that we have

$$
q_{j}^{\gamma}\left(t=t_{m_{j}}\right)=0, \quad t_{m_{j}}=\frac{2}{\beta_{0}} \ln \frac{\alpha_{s}\left(P^{2}\right)}{\alpha_{s}\left(m_{j}^{2}\right)}
$$

for $j=\lambda, s_{L S}, q_{H}, s_{H}$ and $s_{L N S}$. By solving the above coupled equations (3.8) we get the initial condition (3.6).

Now we write down the moments of the structure function in terms of the parton distribution functions and the coefficient functions, which are $\mathscr{O}\left(\alpha_{s}^{0}\right)$ at LO. We take

$$
\vec{C}_{n}^{(0)}(1,0)^{T}=\left(0,0,\left\langle e^{2}\right\rangle_{L},\left\langle e^{2}\right\rangle_{L}, e_{H}^{2}, e_{H}^{2}, 1,1\right)
$$

Then the $n$-th moment of the structure function $F_{2}^{\gamma}$ to the leading order in SQCD is given by

$$
\begin{aligned}
& M_{n}^{\gamma}=\int_{0}^{1} d x x^{n-1} F_{2}^{\gamma} / x=\vec{q}^{\gamma}(n) \cdot \vec{C}_{n}^{\gamma}(1,0) \\
& =\left\langle e^{2}\right\rangle_{L} q_{L S}+\left\langle e^{2}\right\rangle_{L} S_{L S}+e_{H}^{2} q_{H}^{2}+e_{H}^{2} s_{H}+q_{L N S}+s_{L N S} .
\end{aligned}
$$

We have solved the equations (3.8) for $\vec{q}^{\gamma}(n, 0)$ numerically, and plug them into (3.7) for the parton distributions and then evaluate the moments of the structure function $F_{2}^{\gamma}$ by the formula (3.10). By inverting the Mellin moment we get the $F_{2}^{\gamma}$ as a function of $x$. 
In Fig.5, we have plotted our numerical results for the $F_{2}^{\gamma} / \alpha$. The 2dot-dashed (red) and dashed (green) curves correspond to the $F_{2}^{\gamma} / \alpha$ for the massless QCD and SQCD. For the more realistic case, we take $n_{f}=6$ treating the $u, d, s, c$ and $b$ as massless and take the top quark $t$ massive. We assume that all the squarks possess the same heavy mass and the gluino has another heavy mass. In these analyses, we have taken $Q^{2}=(1000)^{2} \mathrm{GeV}^{2}$ and $P^{2}=(10)^{2} \mathrm{GeV}^{2}$. We took the top mass $m_{\text {top }}=175 \mathrm{GeV}$, the common squark mass, $m_{s}=300 \mathrm{GeV}$ and the gluino mass $m_{\lambda}=700 \mathrm{GeV}$.

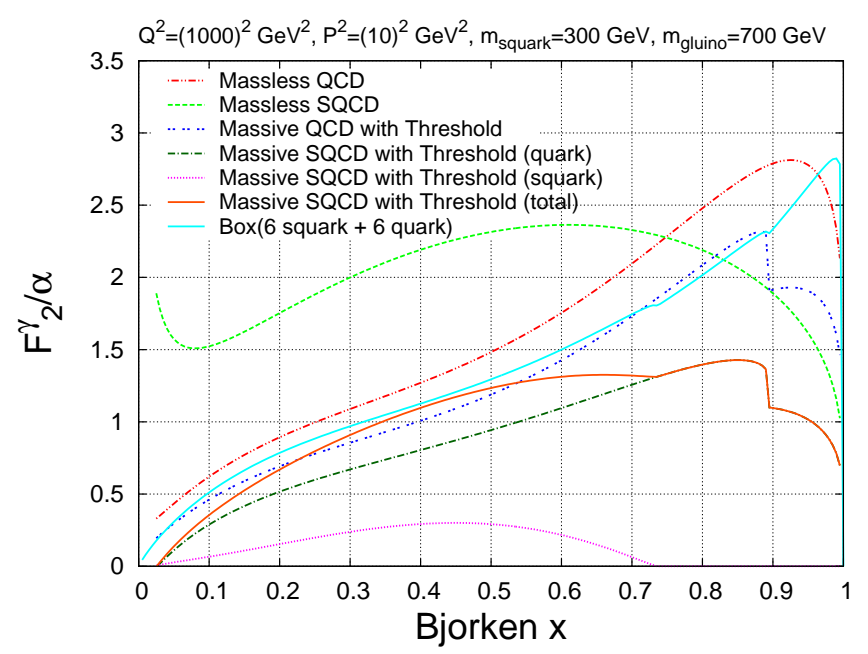

Figure 5: $F_{2}^{\gamma}\left(x, Q^{2}, P^{2}\right) / \alpha$ with SUSY particles as well as top threshold included. The dashed (2dot-dashed) curve corresponds to the massless SQCD (QCD) case. The double-dotted curve shows the massive QCD case. The dash-dotted (dotted) curve corresponds to the quark (squark) component of the massive QCD. The solid curve means the $F_{2}^{\gamma} / \alpha$ for the massive SQCD. The kink at $x=0.89(0.74)$ corresponds to the top (squark) threshold. The light-blue solid curve shows the parton-model result with 6 quarks (heavy top) and 6 massive squarks.

The double-dotted (blue) curve shows $F_{2}^{\gamma} / \alpha$ for the QCD with the mass of the top quark as well as the threshold effects taken into account. The dash-dotted (dark-green) curve shows the quark component for the massive SQCD case with massive top quark, while the dotted (pink) curve means the squark component for the same case. The sum of these leads to the solid (orange) curve which corresponds to $F_{2}^{\gamma} / \alpha$ for the massive SQCD with massive top and threshold effects included. Here, we adopt the prescription for taking into account the threshold effects by rescaling the argument of the distribution function $f(x)$ as $f(x) \rightarrow f\left(x / x_{\max }\right)$ with $x_{\max }$ given in 2.77. After this substitution the range of $x$ becomes $0 \leq x \leq x_{\max }$. In this figure we have also shown the parton-model result with 6 quarks (heavy top) and 6 massive squarks by light-blue solid curve.

At small $x$, there is no significant difference between massless and massive $\mathrm{QCD}$, while there exists a large difference between massless and massive SQCD. At large $x$, the significant masseffects exist both for non-SUSY and SUSY QCD. The SQCD case is seen to be much suppressed at large $x$ compared to the QCD. The squark contribution to the total structure function in massive SQCD appears as a broad bump for $x<x_{\max }$. Here of course we could set the squark mass larger than $300 \mathrm{GeV}$, e.g. around $1 \mathrm{TeV}$, as recently reported by the ATLAS/CMS group at LHC, for higher values of $Q^{2}$. 


\section{Summary}

In this talk we have investigated the photon structure in the SUSY QCD. We first study squark contributions to the eight structure functions of the virtual photon. These structure functions are found to satisfy the positivity constraints and show different behaviors compared to the quark contribution. By setting the suitable boundary conditions for the heavy parton distributions in DGLAP evolution equation we investigated the SQCD radiative effects in the presence of the heavy sparticles. There is no significant difference in the small- $x$ region between QCD and SQCD, while at large $x$, it turns out that there exists a sizable difference between the massive QCD and SQCD. When compared to the squark contribution to $F_{2}^{\gamma}$ in the parton model calculation [15], the squark component in the SQCD is suppressed at large $x$ due to the radiative correction. We expect that the future linear collider would enable such an analysis to be carried out on photon structure functions.

\section{Acknowledgements}

I would like to thank the organizers of RADCOR 2011 for their wonderful organization and hospitalities at this very productive symposium.

\section{References}

[1] T.F. Walsh, Phys. Lett. 36B, 121 (1971); S.J. Brodsky, T. Kinoshita and H. Terazawa, Phys. Rev. Lett. 27 , 280 (1971).

[2] M. Krawczyk, A. Zembrzuski and M. Staszel, Phys. Rept. 345, 265 (2001); R. Nisius, Phys. Rept. 332, 165 (2000); M. Klasen, Rev. Mod. Phys. 74, 1221 (2002); I. Schienbein, Ann. Phys. 301, 128 (2002); R. M. Godbole, Nucl. Phys. Proc. Suppl. 126, 414 (2004).

[3] E. Reya, Phys. Lett. B124 (1983) 424.

[4] D. A. Ross and L. J. Weston, Eur. Phys. JC18 (2001) 593.

[5] M. Drees, M. Glück and E. Reya, Phys. Rev. D30 (1984) 2316.

[6] C. Kounnas and D. A. Ross, Nucl. Phys. B214 (1983) 317.

[7] S. K. Jones and C. H. Llewellyn Smith, Nucl. Phys.B217 (1983) 145.

[8] D. M. Scott, W. J. Stirling, Phys. Rev. D29, 157 (1984).

[9] Y. Kitadono, K. Sasaki, T. Ueda and T. Uematsu, Prog. Theor. Phys. 121 (2009) 495; Phys. Rev. D81 (2010) 074029; Y. Kitadono, Phys. Lett. B702 (2011) 135.

[10] Y. Kitadono, R. Sahara, T. Ueda and T. Uematsu, Eur. Phys. J. C70 (2010) 999.

[11] V. M. Budnev, V. L. Chernyak and I. F. Ginzburg, Nucl. Phys. 34, 470 (1971).

[12] M. Buza, Y. Matiounine, J. Smith, R. Migneron and W. L. van Neerven, Nucl. Phys. B 472 (1996) 611; I. Birenbaum, J. Blümlein and S. Klein, Nucl. Phys. B 780 (2007) 40; 820 (2009) 417.

[13] M. Glück, E. Reya and M. Stratmann, Phys. Rev. D 51 (1995) 3220;D 54 (1996) 5515; F. Cornet, P. Jankowski, M. Krawczyk and A. Lorca, Phys. Rev. D 68 (2003) 014010; F. Cornet, P. Jankowski and M. Krawczyk, Phys. Rev. D 70 (2004) 093004.

[14] K. Sasaki, J. Soffer and T. Uematsu, Phys. Rev. D 66 (2002) 034014.

[15] Y. Kitadono, Y. Yoshida, R. Sahara and T. Uematsu, Phys. Rev. D84 (2011) 074031.

[16] R. Sahara, T. Uematsu and Y. Kitadono, arXiv:1111.4765 [hep-ph] (Nov.2011). 